\title{
A Lower-Pressure Route to Superconductivity
}

\author{
A new synthesis technique pushes high-temperature superconducting \\ materials a step closer to ambient pressure.
}

By Erika K. Carlson

n October 2020, Elliot Snider of the University of Rochester, New York, and colleagues demonstrated room-temperature superconductivity at high pressures. Now, using a new synthesis method, the group has created a material that's superconductive at a similar temperature but at a lower pressure [1]. The new technique could lead to the creation of further novel superconducting materials and thus to new opportunities to test theories of superconductivity.

Like last year's work, in their new demonstration, the researchers used a diamond anvil cell to create a hydrogen-rich compound under high pressure. In the previous experiment, the researchers combined hydrogen with carbon and sulfur. This time they combined the hydrogen with yttrium, a reactive transition metal, to create superconducting yttrium superhydride. Specifically, the researchers placed gaseous hydrogen and solid yttrium between two diamond anvils, with the two materials separated by a thin sheet of palladium. The palladium layer shielded the yttrium and kept it from oxidizing, but it also acted as a catalyst, helping to transport hydrogen

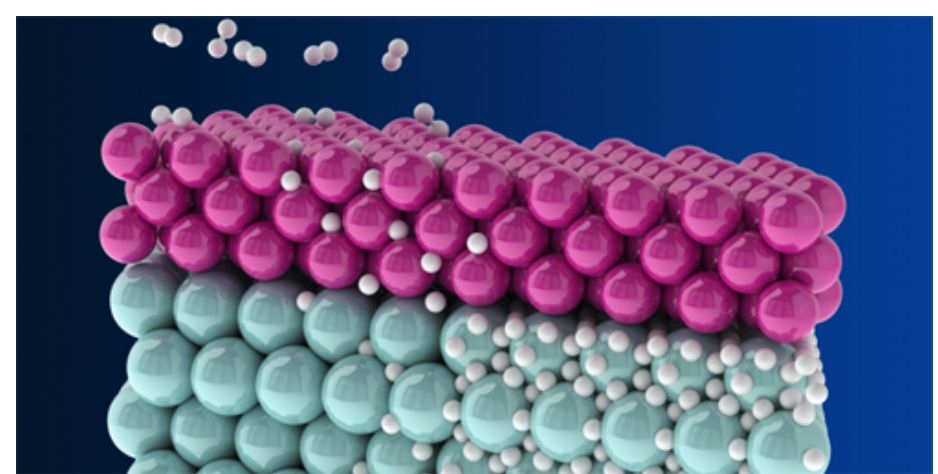

Credit: D. Smith/Argonne National Laboratory; R. Dias/University of Rochester atoms into the yttrium.

A high hydrogen content is thought to promote high-temperature superconductivity, and the new formulation bears this out. The new material is superconductive at about $262 \mathrm{~K}$ and $182 \mathrm{GPa}$ - a significantly lower pressure than the roughly $267 \mathrm{GPa}$ required for last year's room-temperature superconductor, but still much too high for practical applications.

The researchers hope that the technique will allow the synthesis of other high-temperature superconductors, leading to a better understanding of the structures and mechanisms behind the phenomenon. The eventual goal, they say, is to find high-temperature superconductors that work at lower pressures and to develop ways to synthesize them in large quantities.

Erika K. Carlson is a Corresponding Editor for Physics based in New York City.

\section{REFERENCES}

1. E. Snider et al., "Synthesis of yttrium superhydride superconductor with a transition temperature up to $262 \mathrm{~K}$ by catalytic hydrogenation at high pressures," Phys. Rev. Lett. 126, 117003 (2021). 